\title{
Ontological User Modeling for Ambient Assisted Living Service Personalization
}

\author{
Maurício Fontana de Vargas and Carlos Eduardo Pereira \\ Automation Engineering Department \\ Federal University of Rio Grande do Sul \\ Porto Alegre, Brazil \\ mauricio.vargas@ufrgs.br, cpereira@ece.ufrgs.br
}

\begin{abstract}
Given that the population is aging, it is crucial to develop technologies which will not only help the elderly to age in place, but also live in place with independent and healthy lifestyle. Ambient Assisted Living (AAL) environments can help the elderly and people with functional diversity by anticipating their needs in specific situations and acting proactively in order to properly assist them in performing their activities of daily living (ADLs). Since the users needs tend to be very diverse in regard to functioning and disability levels, it is crucial to have personalized services capable of providing tailored assistance to a user based on their unique preferences, requirements, and desires. This paper introduces the ontology named AATUM (Ambient Assistive Technology User Model), to be adopted by systems whose goal is to enhance user quality of life within ALL environments through service personalization. Its main feature is the use of The International Classification of Functioning, Disability and Health (ICF) to model the user's functioning and disability levels in a consistent and internationally comparable way. The use of the proposed ontology is illustrated through its application in two different case studies.
\end{abstract}

Keywords: Ontology, Context-aware, Functioning, User Centered, World Health Organization

\section{Introduction}

The percentage of global population aged 60 or older is expected to be $22 \%$ by 2050 [8]. As a result, there is an expected increase of chronic illnesses and disability associated with old age. This demographic change toward an aging society results in many social and health care system challenges to ensure that our infrastructures can support the needs of the elderly, enabling them to have an independent and healthy lifestyle.

Given the fact that $64 \%$ of older adults prefer to stay in the comfort of their own homes, and given the costs of nursing home care, it is crucial to develop technologies that help older adults not only to age, but also live in place i.e, independently and comfortably in their home [2]. 
In recent years, researchers have developed a variety of assistive technologies based on a the paradigm called "ambient intelligence", where the ambient anticipates users needs in specific situations and acts proactively in order to properly assist the user to perform his/her activities of daily living (ADLs). Assisted living technologies based on ambient intelligence are called Ambient Assisted Living (AAL) tools and can be used for preventing, healing, and improving quality of life of the elderly and people with functional diversity.

Since these users tend to be greatly variable in regards to functioning and disability levels, service personalization is crucial [7]. A personalized service is a service capable of providing tailored assistance to a user based on their unique preferences, requirements, and desires. Therefore, service personalization within AAL environments can help elderly people or people with functional diversity to increase their independence and quality of life.

The need for service personalization has led to the use of ontologies as a means to provide a correct user model in a machine understandable format. This model is generally represented in the form of a user profile which captures the personal aspects in terms of user's behaviors, goals, capacities, likes and dislikes. Therefore, the user model can be seen as an abstract entity and the user profile represents an instantiation of the user model for a specific user [1].

This work focuses on user modeling using an ontology to enhance user quality of life within ALL environments through service personalization. Section 2 presents related work within user modeling and service personalization. The third section presents the Ambient Assistive Technology User Model (AATUM) ontology and its structure, highlighting the main covered aspects and describing its functionality. Then, in the fourth section, two use cases are presented showing real life situations; these are used in the fifth section in order to demonstrate the ontology usage. The last section includes conclusions and future research work.

\section{Related Work}

As mentioned before, one of the core aspects in service personalization is user modeling. Ontology-based user modeling has been previously proposed in many research areas like knowledge management systems [12], semantic web search [6] and digital museum guides [4]. The increasing attention of ontological modeling is mostly due to its interoperability feature and ability to enable knowledge sharing and reuse over several application domains [5].

One important issue is that user preference and needs may change depending on the user context. Thus, the system has to be able to infer which context the user is in and consequently adapt the ambient to provide the appropriate assistance. The UPOS (User Profile Ontology with Situation-Dependent Preferences Support) [16] introduced the concept of dynamic user profiles. The ontology supports the creation of a subset of conditional user profiles associated with the user context. According to a condition (e.g. if the context of user Bob equals the MyOffice location), the matching process tries to find the correspondent sub-profile. If a match happens, the found user profile is applied for service personalization. 
Another relevant concern within AAL service personalization is the need of having information about the user's health condition and his/her limitations performing daily activities. Without the correct representation of the user's needs and capabilities, it's unlikely that the ambient will properly adapt itself to provide optimum assistance for the elderly and for people with special requirements.

R. Kadouche et al. [7] proposed the Semantic Matching Framework (SMF) capable of providing an appropriate middleware for delivering personalized assistive services according to the user's needs and capabilities. The main feature of SMF is based on the semantic matching between the user model and the environment model. The user model describes user information, preferences and capabilities while the environment model defines the available devices (e.g. door) and their attributes (e.g. required force to open the door). Using a reasoning mechanism, the SMF analyzes user capabilities and the environment and deduces the "handicap situation" in order to deliver personalized services.

The PCEICL (Person Centered Environment for Information, Communication and Learning) [3] platform aims to offer a better assistance to elderly people using context aware and personalizable services. They have proposed an ontology where the user is the central concept and is described by their characteristics such as their health condition, capabilities and preferences. In order to have an exact and correct description of the user's health condition, they have used the International Code for Diseases (ICD) [11].

Another approach of service personalization in the field of AAL is the MobileSage [15]. The main purpose of their work is to provide help on-demand services as the user moves between mobile environments. Based on the user's characteristics and his/her location, the system can personalize services in order to assist the user in his/her daily activities like outdoor navigation and the use of devices such as ticket vending machines. As in [3], MobileSage ontology models the user as a central concept of the system as well as his/her environment. The user profile is composed by a set of sub profiles like a capability profile, a health profile and an interest profile.

The main issue of the aforementioned works is the lack of a uniform and consistent representation of user health condition, functioning and disability. The representation of this information is usually designed in order to meet the requirements of a specific application. Thus, the main feature of using an ontology, i.e. the knowledge sharing and reuse, is not fully utilized.

\section{The AATUM ontology}

In order to prevent common mistakes and make the best modeling decisions while developing the ontology, we have taken into account the design method proposed by [9]. Using a traditional top-down approach, both static and dynamic highlevel concepts related to the user were selected and further broken down into specialized concepts. The AATUM ontology has been implemented in OWL, the Ontology Web Language that is a xml-based semantic web language proposed by the World Wide Web Consortium (W3C). To help with the process of creating, 
editing and viewing the ontology, the Protégé-OWL Editor ${ }^{1}$ was selected. A brief explanation of the ontology main classes is depicted in Table 1. To illustrate the ontological structure and the relationship between its classes, a graphical representation is presented in Fig. 1.

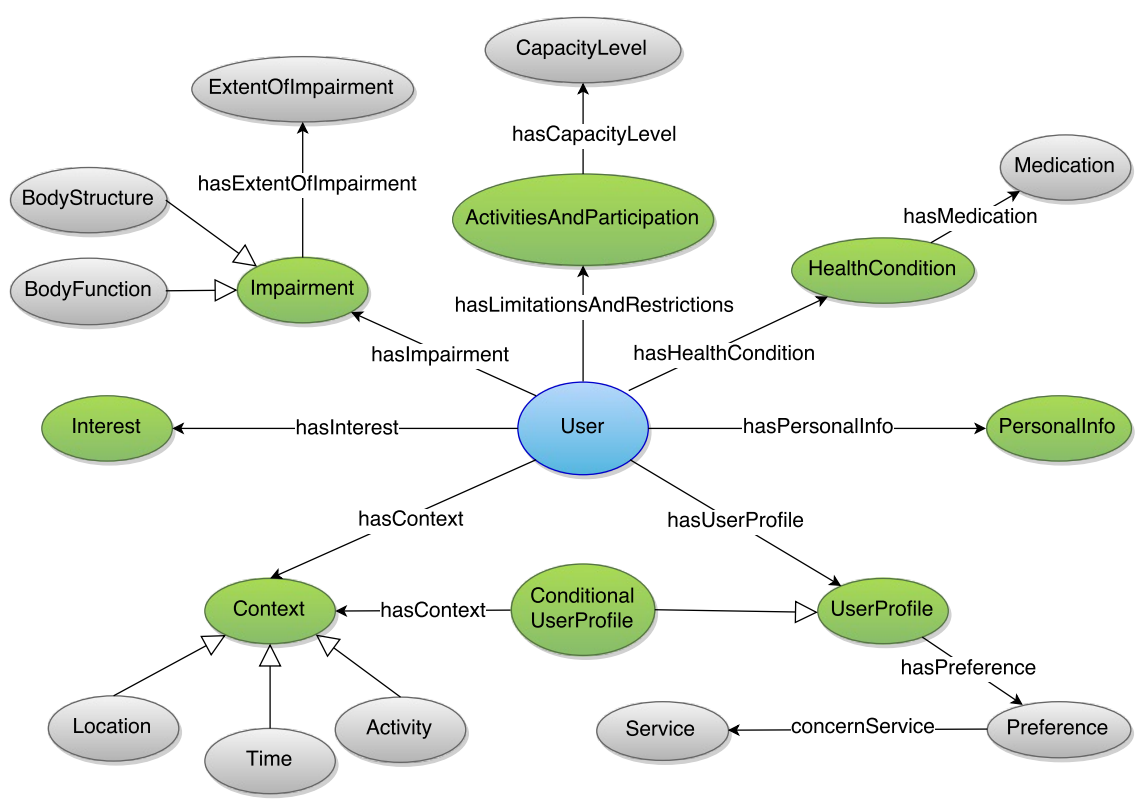

Fig. 1. An overview of the AATUM ontological structure

Basic information such as address, date of birth, telephone number and family contacts is stored in the class PersonalInfo. Though this information may not seem to be very useful, it could be used for important purposes in terms of ALL. For example, this information could be used to send an ambulance to a user's house after a heart attack or in a statistical report documenting the health conditions of a neighborhood's residents.

The class Interest holds information about personal interests related either to a hobby or work. Every interest has a weight associated in order to measure how much the user is interested in that specific subject. Moreover, additional information like descriptions or schedules can be stored. This class is useful for recommendations or content-filtering applications and can help people to have a higher quality of life. For example, the task of choosing a movie on television would be much easier for an elderly person if only desirable options were presented.

\footnotetext{
${ }^{1}$ Protégé-OWL Editor: Available at http://protege.stanford.edu/
} 
Table 1. AATUM ontology main classes

\begin{tabular}{l|l|l}
\hline Concept & Description & Example Values \\
\hline \hline User & The user of the system & "User_John" \\
\hline User Profile & $\begin{array}{l}\text { Default profile associated to } \\
\text { preferences of one particular user }\end{array}$ & "John_DefaultProfile" \\
\hline $\begin{array}{l}\text { User Conditional } \\
\text { Profile }\end{array}$ & $\begin{array}{l}\text { Conditional profile associated to } \\
\text { the user's context describing } \\
\text { situation-dependent preferences. }\end{array}$ & "John_SleepingProfile", \\
"Noel_LivingRoomProfile",
\end{tabular}


The class Context is used to represent user's context such as the location, the time, and the activity the user is performing. One key advantage of defining the context this way is the possibility of inferring additional information from a small amount of sensor data. For example, using only the information that the user is located in his bedroom and it is 01:00 AM, the system can infer that the user is sleeping and then adapt the ambient in order to give the user a better night sleep.

The user's behavior and preferences depend on the temporal and environmental context the user is in. For example, the user's smartphone should be set to silent mode during a meeting and to loud mode when the user is at home; the shower water temperature is likely to be higher in the winter compared to the summer; the desired ambient luminosity may be set to high in the evening and to none during sleep time. Thus, it's crucial to have different profiles related to different contexts. This is done through the class ConditionalUserProfile that is linked to the class Context. The preferences that are unlikely to change according to the context are linked to a default profile represented by the class UserProfile, which is the superclass of ConditionalUserProfile .

The most important concepts of the ontology are the classes related to the user's health condition and capabilities. Having a complete, uniform, and consistent model of the user's functioning and disability levels allows correct service tailoring, and thus a better quality of life for impaired and elderly people.

Information about user health condition, i.e, the disease or disorder diagnosis is stored in HealthCondition class. As in [3], this information is composed by the disease/disorder level, its related medication, and its ICD, which is a worldwide used code. Also, additional information relevant to medical accompaniment or treatment can be stored. This information could be used, for instance, to give an analysis of the general health situation of a population group or to prompt the user about his/her medication schedule.

According to [10], diagnosis of a disease or disorder by itself does not predict service needs, length of hospitalizations, level of care, or functional outcomes. If a person cannot perform an activity it may be related to any of various different health conditions. In other words, it is very hard to infer participation in everyday life from medical diagnosis alone. This implies that without data about levels of functioning and disability, we will not have the information needed to properly assist the user in his daily activities. Therefore, we used The International Classification of Functioning, Disability and Health (ICF) to model the user's functioning and disability levels in a consistent and internationally comparable way. An excerpt of the class describing user impairments is shown in Fig. 2 while Fig. 3 presents an excerpt of the class that describes user limitations performing activities and participation restrictions.

We also have used the ICF qualifiers to record the presence and severity of a problem in functioning at the body, person and societal levels. For the classifications of body function and structure, the qualifier Extent0f Impairment indicates, on a five point scale, the degree of the impairment of function or structure. The possible values are: no impairment, mild, moderate, severe and 
complete. In the case of the Activity and Participation list of domains, the Capacity qualifier describes an individual's ability to execute a task or an action using the same five point scale.

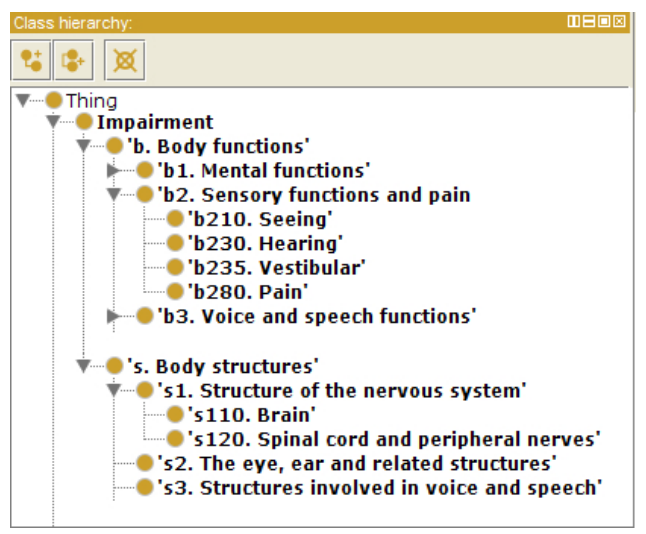

Fig. 2. An excerpt of the Impairment class as shown within Protégé

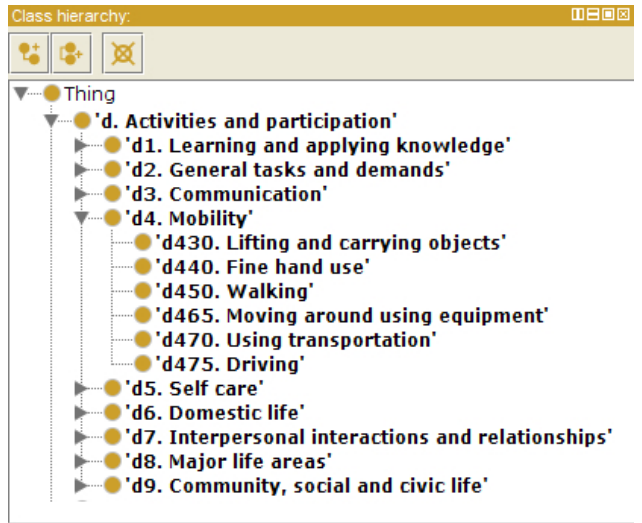

Fig. 3. An excerpt of the ActivitiesAndParticipation class as shown within Protégé

\section{Use case study}

Based on the elderly and people with functional diversity most important requirements presented in [13] and [14], two use cases where the ontology can be 
used in order to properly assist the user to perform his/her activities of daily living were defined. First, a short description of the users is given:

Mr. John: Mr. John is a 63 year old retired Professor living alone is his apartment. Though he left his job recently, he still reads scientific news on his personal computer or tablet. He started to cook as a hobby and a few weeks ago he started to follow a foreign documentary about Asian cuisine that is broadcast every Wednesday night. He has a high level of myopia which makes it hard to read small or distant texts.

Mr. Noel: Mr. Noel is a 81 year old widower living with his daughter. Because he suffers from cardiac arrhythmia, his heart rate needs to be checked constantly. His daughter used to assist him with the daily care needs such as giving the medicines and checking his heart rate, but since she got a new job, Mr. Noel will have to stay by himself during weekday afternoons.

Use case 1: Every Wednesday night the system will show a notification in Mr. John's smartphone telling him that the documentary is about to start. Moreover, additional information about the specific episode is shown. If Mr. John confirms he's going to watch the documentary, the system will turn on the TV, switch to the correct channel, set the subtitles to English and adjust its size in order to make it possible for Mr John to read it comfortably. Also, the room lights are turned off.

Use case 2: While Mr. Noel is by himself at home, his heart rate is obtained by a wearable sensor and transmitted wirelessly to the system. This data is compared with values determined safe by his doctor, and in case of an emergency, both his daughter and medical staff are notified. Since Mr. Noel takes two different medications, the system attends to his medicine schedule and prompts him when medication is required. This is done through the closest electronic device, such as the living room television or his personal computer.

\section{Ontology usage}

Once we have the user model ontology, we need a infrastructure capable of delivering customizable services available in the ambient. Fig. 4 presents an overview of a service-oriented architecture (SOA) within a smart home that aims to assist the user with special requirements to have a safe and independent lifestyle using a combination of context-awareness, user modeling and service personalization. This architecture is part of our ongoing research and will be further discussed in future papers.

The data needed for populating the ontological user model is gathered in several ways. Context invariant data such as personal information, interests and health condition is acquired during the system initialization by the user or the caregiver. On the other hand, information such as preferences can be deduced from previously given information. For example, if the user has a mild hearing impairment, his preference for volume level should automatically be set to high.

Specifically for collecting the health and disability information, the system can use one of the two application instruments proposed by the World Health 


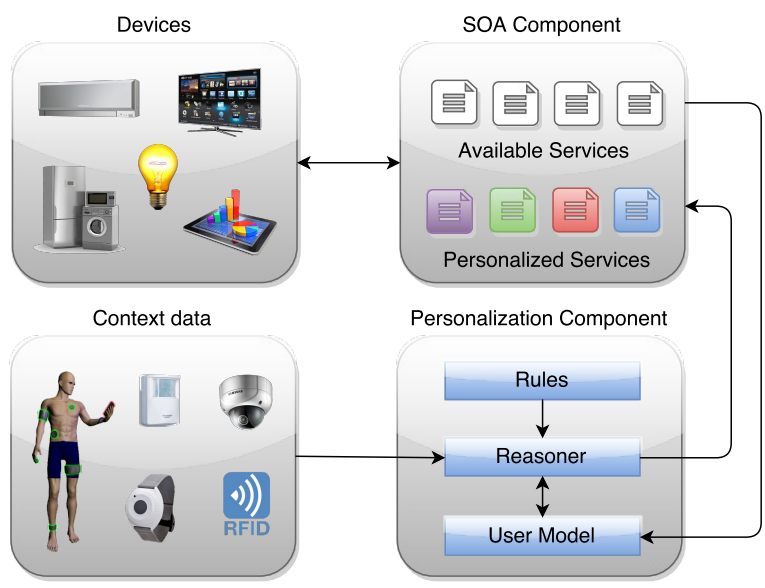

Fig. 4. System architecture for service personalization in a smart home

Organization (WHO). This is another compelling benefit of using the ICF as part of the ontology. The first one is a checklist with the most relevant ICF categories which allows the identification and qualifications of the individuals functioning profile in a straightforward manner. The second one, named WHODAS (WHO Disability Assessment Schedule), is a generic assessment instrument designed from a comprehensive set of the ICF items that are sufficiently reliable and precise for providing a standardized method for measuring health and disability across different cultures [17]. A unique feature of WHODAS, that distinguishes it from other disability measures, is its direct association to the ICF. According to the WHO, the WHODAS 12-item version has an average interview time of only five minutes, what it makes it a very affordable strategy to collect all necessary user's health and disability information.

The next subsections depict how the proposed AATUM ontology and the system architecture are used to provide user optimal assistance with the use cases introduced in Section 4.

\subsection{Use case 1}

Mr. John's interest about the Asian cuisine documentary is stored as an instance of the class Interest and the documentary's hours and channel is saved as the class's property AdditionalInformation.

Knowing the current day and time, the system searches on the Internet additional information about the episode and sends to John's smartphone. After Mr. John confirms he's going to watch the episode, the living room television is turned on and set to the documentary's channel. 
Using the information about his seeing impairment stored as an instance of the ICF class b210. Seeing along his context information Watching TV, the system infers the preference Subtitle size: large and saves it as an instance of the class Preference that is linked to his conditional profile John_WatchingTvProfile.

In the last times Mr. John watched TV, he turned off the room's lights. This pattern was learned by the system reasoner using a predefined rule and stored as the preference Room lightning: dark that is also linked to his conditional profile John_WatchingTvProfile.

An excerpt of the ontology classes instances describing this use case is presented in Fig. 5.

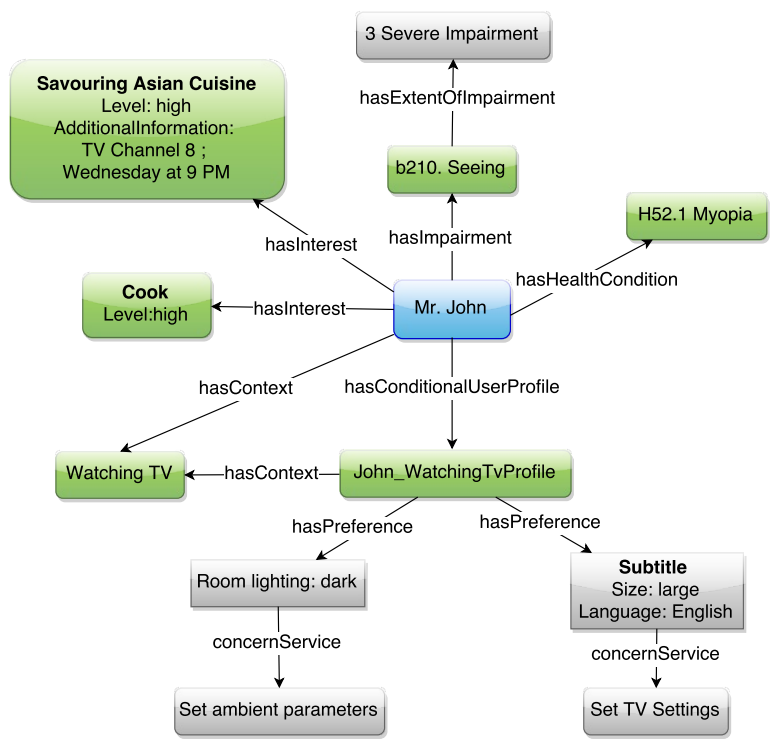

Fig. 5. Ontology classes instances for Use Case 1

\subsection{Use case 2}

Mr. Noel's heart disease is stored as an instance of the class HealthCondition, as it's ICD code (I49.9) and medication schedule. The heart rate values determined safe by his doctor are stored as the class' property AdditionalInformation. His daughter's and hospital's telephone number, as his address, are saved in the class PersonalInfo. This way, when his body sensor detect unsafe values, the system sends a text message notifying his daughter and calls an ambulance to his house. After attending his medicine schedule, the system determines the device where the notification should be displayed using his context information stored in Location, that was obtained through presence sensors. 


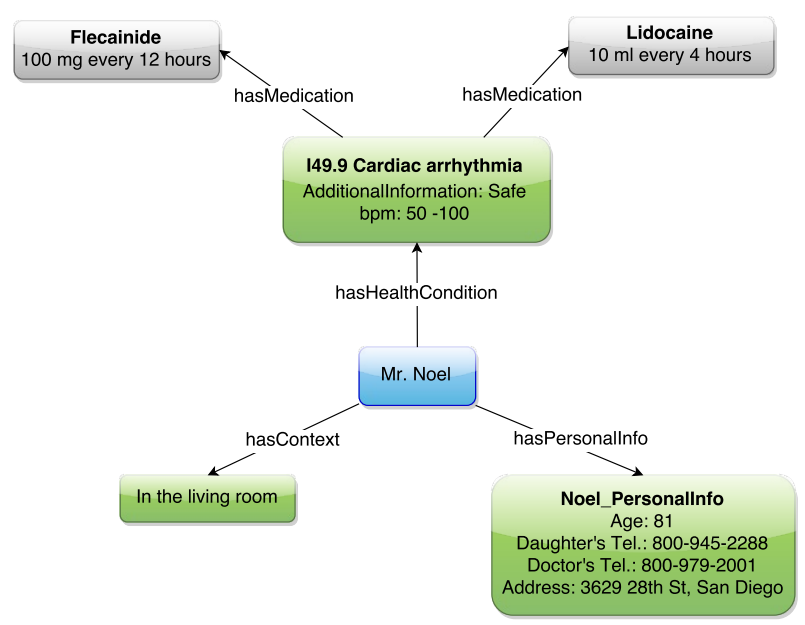

Fig. 6. Ontology classes instances for Use Case 2

An excerpt of the ontology classes instances describing this use case is presented in Fig. 6.

\section{Conclusion}

In this paper we have presented the AATUM, a novel ontology for user modeling in the field of AAL. We have demonstrated, through two case studies, how the proposed ontology is used to properly assist the elderly with their activities of daily living in a smart home, achieving a safe and independent lifestyle.

The use of ontologies for user modeling within AAL is not new, but the related works fail in the representation of user health condition, functioning, and disability, which are essential components to properly provide optimum assistance for the elderly and for people with special requirements. In the AATUM ontology, the user is the central concept and is described by his/her static and dynamic properties such as personal information, interests, health condition, etc. The AATUM ontology main feature is the use of the ICF, the World Health Organization's framework for health and disability, to model the user's functioning and disability levels in a consistent and internationally comparable way. Also, the ICD code is used to properly describe the disease or disorder diagnosis. Another compelling feature is the use of a conditional user profile related to the user's context to describe situation-dependent preferences.

Future work will aim to develop the reasoning mechanism to provide personalized services according to the user profile described by the AATUM ontology and his/her context. Further evaluation will involve the total implementation and integration of the service personalization component with a smart home 
based on a service-oriented and context-aware architecture.

Acknowledgment. This research work has been funded by the CAPES PROCAD project $(071 / 2013)$, whose support is gratefully acknowledged.

\section{References}

1. Bhowmick, P.K., Sarkar, S., Basu, A.: Ontology based user modeling for personalized information access. IJCSA 7(1), 1-22 (2010)

2. for Disease Control, C., (CDC), P., et al.: The state of aging and health in america 2013. Atlanta, GA: Centers for Disease Control and Prevention, US Department of Health and Human Services (2013)

3. Fredrich, C., Kuijs, H., Reich, C.: An ontology for user profile modelling in the field of ambient assisted living. In: SERVICE COMPUTATION 2014, The Sixth International Conferences on Advanced Service Computing. pp. 24-31 (2014)

4. Hatala, M., Wakkary, R.: Ontology-based user modeling in an augmented audio reality system for museums. User Modeling and User-Adapted Interaction 15(3-4), 339-380 (2005)

5. Heckmann, D., Schwartz, T., Brandherm, B., Schmitz, M., von WilamowitzMoellendorff, M.: Gumo-the general user model ontology. In: User modeling 2005, pp. 428-432. Springer (2005)

6. Jiang, X., Tan, A.H.: Learning and inferencing in user ontology for personalized semantic web search. Information sciences 179(16), 2794-2808 (2009)

7. Kadouche, R., Mokhtari, M., Giroux, S., Abdulrazak, B.: Personalization in smart homes for disabled people. In: Future Generation Communication and Networking, 2008. FGCN'08. Second International Conference on. vol. 2, pp. 411-415. IEEE (2008)

8. Lutz, W., Sanderson, W., Scherbov, S.: The coming acceleration of global population ageing. Nature 451(7179), 716-719 (2008)

9. Noy, N.F., McGuinness, D.L., et al.: Ontology development 101: A guide to creating your first ontology (2001)

10. Organization, W.H.: International classification of functioning, disability and health: ICF. World Health Organization (2001)

11. Organization, W.H., et al.: International classification of diseases (icd) (2012), http://www . who.int/classifications/icd/en/, [Retrieved June, 2015]

12. Razmerita, L., Angehrn, A., Maedche, A.: Ontology-based user modeling for knowledge management systems. In: User Modeling 2003, pp. 213-217. Springer (2003)

13. Rusu, L., Cramariuc, B.: A conceptual approach for innovative home care solution. Journal of Applied Computer Science \& Mathematics 17(17), 22-26 (2014)

14. de Ruyter, B., Zwartkruis-Pelgrim, E., Aarts, E.: Ambient assisted living research in the carelab. GeroPsych: The Journal of Gerontopsychology and Geriatric Psychiatry 23(2), 115 (2010)

15. Skillen, K.L., Chen, L., Nugent, C.D., Donnelly, M.P., Burns, W., Solheim, I.: Ontological user profile modeling for context-aware application personalization. In: Ubiquitous Computing and Ambient Intelligence, pp. 261-268. Springer (2012)

16. Sutterer, M., Droegehorn, O., David, K.: Upos: User profile ontology with situationdependent preferences support. In: Advances in Computer-Human Interaction, 2008 First International Conference On. pp. 230-235. IEEE (2008)

17. Üstün, T.B.: Measuring health and disability: manual for WHO disability assessment schedule WHODAS 2.0. World Health Organization (2010) 